\title{
Distinct Gene Expression Pathways in Islets from Individuals with Short- and Long- Duration Type 1 Diabetes
}

Teresa L. Mastracci ${ }^{1,2 *}$, Jean-Valery Turatsinze ${ }^{3}$, Benita K. Book ${ }^{4}$, Ivan A. Restrepo ${ }^{5}$, Michael J. Pugia $^{1}$, Eric A. Wiebke ${ }^{4}$, Mark D. Pescovitz ${ }^{4}$, Decio L. Eizirik ${ }^{3}$ and Raghavendra G.

$$
\text { Mirmira }^{2,5,6,7}
$$

${ }^{1}$ Indiana Biosciences Research Institute, Indianapolis, IN, USA.

${ }^{2}$ Department of Biochemistry and Molecular Biology, Indiana University School of Medicine, Indianapolis, IN, USA.

${ }^{3}$ ULB Center for Diabetes Research, Universite Libre de Bruxelles, Brussels, Belgium.

${ }^{4}$ Department of Surgery, Indiana University School of Medicine, Indianapolis, IN, USA.

${ }^{5}$ Center for Diabetes and Metabolic Diseases, Indiana University School of Medicine, Indianapolis, IN, USA.

${ }^{6}$ Department of Pediatrics, Indiana University School of Medicine, Indianapolis, IN, USA.

${ }^{7}$ Department of Medicine, Indiana University School of Medicine, Indianapolis, IN, USA.

* Corresponding Author: Teresa L. Mastracci, 1345 W. $16^{\text {th }}$ St., Suite 300, Indianapolis, IN 46202 USA. E-mail: tmastracci@indianabiosciences.org

Running Title: Functional Genomics Analysis of T1D Islets

Abstract word count: 272

This is the author's manuscript of the article published in final edited form as:

Mastracci, T. L., Turatsinze, J.-V., Book, B. K., Restrepo, I. A., Pugia, M. J., Wiebke, E. A., ... Mirmira, R. G. (n.d.). Distinct Gene Expression Pathways in Islets from Individuals with Short- and Long-Duration Type 1 Diabetes. Diabetes, Obesity and Metabolism. https://doi.org/10.1111/dom.13298 
Main text word count: 2730

References: 27; Tables: 2; Figures: 3

\begin{abstract}
Aims: Our current understanding of the pathogenesis of type 1 diabetes (T1D) arose in large part from studies using the non-obese diabetic (NOD) mouse model. In the present study, we chose a human-focused method to investigate T1D disease mechanisms and potential targets for therapeutic intervention by directly analyzing human donor pancreatic islets from individuals with T1D.

Materials and Methods: We obtained islets from a young individual with T1D for three years and an older individual with T1D for 27 years and performed unbiased functional genomic analysis by high-depth RNA sequencing; the T1D islets were compared with islets isolated from three non-diabetic donors.

Results: The islets procured from these T1D donors represent a unique opportunity to identify gene expression changes in islets after significantly different disease duration. Data analysis identified several inflammatory pathways upregulated in short-duration disease, which notably included many components of innate immunity. As proof of concept for translation, one of the pathways, governed by IL-23(p19), was selected for further study in NOD mice because of ongoing human trials of biologics against this target for different indications. A mouse
\end{abstract}


monoclonal antibody directed against IL-23(p19) when administered to NOD mice resulted in a significant reduction in diabetes incidence.

Conclusion: While sample size for this study is small, our data demonstrates that the direct analysis of human islets provides a greater understanding of human disease. These data, together with the analysis of an expanded cohort to be obtained by future collaborative efforts, might result in the identification of promising novel targets for translation into effective therapeutic interventions for human T1D, with the added benefit to repurpose known biologicals for use in different indications.

\section{Introduction}

Type 1 diabetes (T1D) is an autoimmune disorder in which autoreactive T cells target and destroy pancreatic insulin-producing beta cells. This is probably preceded by local inflammation and activation of the innate immune response ${ }^{1}$. Much of our current understanding of T1D pathogenesis arose from studies in non-obese diabetic (NOD) mice ${ }^{2}$. Studies performed in animal models do provide researchers with the ability to manipulate the genetics and physiology of a mammalian system, thereby providing valuable information regarding disease pathogenesis and progression. That said, interventions based on pathways identified to be specifically altered in NOD mice ${ }^{3,4}$ have up to now failed in translation to human disease, leading at best to partial stabilization of the insulin secretory capacity of beta cells for short periods of time ${ }^{5,6}$. Additionally, insulitis is not a uniform pathological feature of the pancreas in human $\mathrm{T}^{\mathrm{D}}{ }^{7}$ as it 
is in NOD mice, and the presence of beta cell mass is evident in many individuals years after onset $^{8}$. Therefore, the direct study of human T1D at various stages of progression may better identify targets that reduce progression or reverse disease. The subsequent manipulation or validation of these targets in mouse models would then provide additional support for moving a candidate toward human clinical study.

There has been increasing evidence recently that beta cells may not be simply bystanders in an autoimmune process, but rather active players in the progression and pathophysiology of disease $^{9-11}$. This suggests that an in-depth molecular analysis of human islets from individuals with T1D compared with healthy controls could provide insight into new therapeutic approaches. Here, we present a proof of concept study wherein RNA deep sequencing data from the islets of two individuals with different T1D disease duration were analyzed to parse the changes in gene expression pathways after three years of disease (hereafter referred to as "short-duration") compared with 27 years of disease (hereafter referred to as "long-duration") as compared to no disease (non-diabetic control donors). Our data suggest that disease duration impacts the nature of inflammatory pathways activated, and at least one pathway (Th1/Th17) displays the potential to be exploited in both humans and NOD mice for the prevention or possible remission of diabetes.

\section{Materials and Methods}

Human Islets 
Human islets were obtained from deceased organ donors through the Integrated Islet Distribution Program and Vanderbilt University (https://iidp.coh.org/Centers/Policies-StandardOperating-Procedures). Supplemental Tables 1 and 2 outline donor characteristics. Islet isolations from young donors or donors with long-standing T1D are not a common occurrence and therefore represent an opportunity to analyze unique aspects of the disease. T1D islets were recovered with good viability ( $87 \%$ and $92 \%$, respectively) and purity $(\sim 80 \%)$; islets recovered from three non-diabetic control donors also showed good viability $(95-96 \%)$ and purity ( $80-$ 90\%). Shipments of islets were received at the Indiana University School of Medicine (IUSM) and placed into human islet media, which included standard islet media (Prodo Laboratories), human AB serum supplement (Prodo Laboratories), glutamine and glutathione supplement (Prodo Laboratories) and ciprofloxacin (Fisher). All islets were hand-picked to exclude non-islet material and increase purity. RNA was extracted from 200- 600 islets using the RNeasy Mini Extraction kit (Qiagen) and equivalent concentrations of RNA from each sample were used for RNA sequencing experiments.

\section{RNA Sequencing}

High-depth RNA sequencing was performed using an Illumina HISeq3000 (Indiana University; https://cgb.indiana.edu/sequencing/index.html), with approximately 239 million paired-end reads obtained per sample. Reads were mapped to the human reference genome GRCh37/hg19 using Tophat $2^{12,13}$ and Gencode version 18 annotation dataset ${ }^{14,15}$. Mapped reads were used to 
quantify gene abundance using the FluxCapacitor program version $1.6 .1^{16}$. All genes were assigned a relative expression level measured in RPKM units ('reads per kilobase per million mapped reads') ${ }^{17}$. The two islet samples from T1D donors were analyzed and compared separately with control islets. Genes with expression of RPKM $>0.5$ in at least one of the samples studied and with potential pathogenic relevance were identified and reported in Results. To define a gene as up- or down-regulated, a cut-off was used of a three-fold change (FC) compared to the control median expression value. The association of up- or down-regulated genes with molecular/cellular functions and pathways was performed using Ingenuity Pathway Analysis (IPA; Ingenuity Systems). Raw data was deposited in the Gene Expression Omnibus (GEO) database (GSE102371) and on the IBRI website (http://data.indianabiosciences.org). Gene expression patterns from all T1D and control donor islets were also compared with RNA sequencing data from cytokine-treated human islets (unpublished dataset and ${ }^{18}$ ) as well as other human tissues from the Illumina bodyMap2 transcriptome project available in GEO (GSE30611).

\section{Immunofluorescence}

Pancreatic tissue sections from paraffin-embedded blocks were obtained from the individuals with short-duration T1D, long-duration T1D, and one non-diabetic control. Immunofluorescent staining was performed as previously published ${ }^{19}$. Primary antibodies used include guinea pig anti-insulin (1:1000; Thermo), mouse anti-glucagon (1:500; Abcam), mouse 
anti-CD66b conjugated with DyLight 550 (1:200; clone G10F5; Novus), rabbit anti-amylase (1:500; Sigma). For all non-conjugated primary antibodies, Alexa-488, Cy3, and Alexa-647 secondary antibodies (1:500; Jackson ImmunoResearch) were then used. DAPI (Sigma) was used to visualize nuclei. Images were acquired with a Zeiss 710 confocal microscope.

\section{Drug studies in NOD mice}

Female NOD mice were obtained from Jackson Laboratories (Bar Harbor, ME) at 5 weeks of age and acclimated for 1 week prior to experimentation. All animal studies were approved by the Institutional Animal Care and Use Committee at IUSM. Anti-IL-23(p19) (CNTO6163) and mouse IgG isotype control (CNTO1322) were provided by Jansen Research and Development, LLC. Drugs were administered by intraperitoneal injection to animals at 6 weeks of age. To determine treatment outcome, blood was drawn weekly to assess glycemic control. Diabetes was defined as blood glucose $>250 \mathrm{mg} / \mathrm{dL}$ on three separate occasions; after the first value $>250 \mathrm{mg} / \mathrm{dL}$, daily monitoring was performed. The treatment groups were: (1) 6week-old NOD mice treated weekly for 27 weeks with $0.5 \mathrm{mg} / \mathrm{mouse}$ CNTO6163 in PBS

(N=24); (2) 6-week-old NOD mice treated weekly for 27 weeks with $0.5 \mathrm{mg} / \mathrm{mouse}$ CNTO1322 in PBS $(\mathrm{N}=12)$. Pancreas tissue was collected for insulitis scoring, which was performed as described previously ${ }^{20}$.

\section{Results}




\section{Differential gene expression in human islets from individuals with T1D}

Using RNA-sequencing, we characterized differential gene expression in islets isolated from two individuals with T1D of vastly different duration. The individual designated as shortduration was 6 years old and had T1D for 3 years whereas the individual designated as longduration was 38 years old and had T1D for 27 years. For comparison, we profiled islets from three non-diabetic individuals, aged 11, 12, and 64 (Supplemental Tables 1 and 2). Gene expression patterns from all islet samples were compared with gene expression data from other human tissues collected as part of the Illumina bodyMap2 transcriptome project (Figure 1). Importantly, islet preparations clustered together but were separate from other tissues, indicating that even with diabetes the islets retained an "islet identity signature".

Short-duration T1D islets showed 667 upregulated and 2564 downregulated genes compared with controls. Similarly, comparing long-duration T1D with controls identified 660 upregulated and 2539 downregulated genes. Despite a similar total number of genes differentially expressed in the islets of both T1D donors (3231 versus 3199 genes), the specific genes altered were not identical, with only $18.1 \%$ and $46.5 \%$ of the up- and down-regulated genes, respectively, overlapping between short- and long-duration T1D.

Expression values for a select group of genes are in Table 1. Of note, insulin (INS) expression is present in T1D islets, but at $<0.1 \%$ of control values. A reduction of insulinexpressing cells was confirmed by immunofluorescence on tissue from these individuals;

glucagon-expressing cells identified islet areas (Supplemental Figure 1). Importantly, this 
immunofluorescence analysis was performed on sections from only one area of the pancreas and therefore can only provide a cellular view of the tissue from that area. The gene expression data gathered by RNA sequencing, on the other hand, provides a more complete picture of islet hormone gene expression across all obtained islets. Interestingly, in addition to the global reduction in insulin gene expression, the expression of all islet hormone genes, including glucagon (GCG), ghrelin (GHRL), somatostatin (SST), and pancreatic polypeptide (PPY), was reduced in both T1D samples compared with controls. Select transcription factors significant to beta cell function, such as MAFA, NEURODI and PDXI, also showed reduced expression (Table 1). Together, these data confirm a reduction in insulin-expressing beta cells and a possible reduction in differentiated islets cells in both individuals with T1D.

\section{Pathways altered in T1D}

To obtain an unbiased global view of the data, we identified pathways corresponding to collections of genes altered in short-duration or long-duration T1D compared with controls (Table 2; Supplemental Figure 2; Supplemental Tables 3-6). Based on the genes and pathways identified, the software also delineated potential upstream regulators of the up- and downregulated pathways (Supplemental Tables 7 and 8). Given the reduction in beta cells observed and quantitative loss of hormone gene expression, these downregulated pathways are likely influenced by altered islet composition. 


\section{Inflammatory genes up-regulated in islets early in disease}

The scale of upregulation [log (B-H p value)] for pathways altered in short-duration T1D was particularly noteworthy and included marked expression of inflammatory-related pathways (Figure 2). "Granulocyte adhesion and diapedesis" was the top canonical pathway increased in short-duration T1D (Table 2). Additionally, several genes usually expressed in neutrophils, such as CXCL1, CXCL2, CXCL3, CXCL5, CXCL10, CXCL12, CCCL12, CCL3 and CCCL20, and the cytokines IL-1 and IL-8, showed much higher expression in short-duration T1D compared with controls (Table 1). These findings suggest there may exist a protracted infiltration into the islets by innate immune cells such as granulocytes or antigen presenting cells (monocytes/macrophages), along with local production of pro-inflammatory cytokines. To test this hypothesis we compared the present T1D islet gene expression findings against our previous findings with human islets exposed to the inflammatory cytokines IL-1beta and IFN-gamma ${ }^{18}$. Interestingly, short-duration T1D islets showed a similarity of 36\% up-regulated (240 mRNAs) and 37\% down-regulated (956 mRNAs) genes compared with cytokine-treated islets; these values were lower in long-duration T1D, namely a similarity of $11 \%$ up-regulated (77 mRNAs) and 29\% down-regulated (745 mRNAs) genes compared with cytokine-treated human islets. In line with this, pathway analysis identified TNF-alpha, IL-1beta, IFN-gamma and their downstream transcription factors NF-kB and STAT $1{ }^{10}$, as key upstream regulators of the gene networks observed as up-regulated in short-duration T1D (Supplemental Table 7). On the other hand, the upstream regulators of the down-regulated genes were transcription factors related to 
beta cell function (Supplemental Table 8), which is in line with the decrease in insulin expression and beta cells (Table 1; Supplemental Figure 1).

\section{Reduced progression to diabetes with early inhibition of IL-23/p19 in NOD mice}

Given the number of inflammatory pathways altered with short-duration T1D, we asked if inhibiting one inflammatory pathway not previously implicated in T1D progression might alter diabetes outcome. Our data identified interleukin 23 alpha subunit p19 (IL23A) and two IL-17A pathways (downstream of IL-23) were increased in short-duration T1D, but not long-duration T1D (Table 1). This target also proved interesting given its current evaluation as a therapeutic for other human autoimmune-related indications, specifically Psoriasis, Crohn's Disease, Plaque Psoriasis, and Ulcerative Colitis (NCT01947933, NCT02891226, NCT02899988, and NCT02589665). We therefore sought to inhibit IL-23(p19) in NOD mice early in the course of disease (6 weeks), corresponding to the early stages of insulitis (Figure 3A). NOD mice treated at 6 weeks of age with anti-IL-23(p19) were followed with weekly blood glucose measurements to determine diabetes onset. Interestingly, treated mice showed significantly reduced progression to diabetes compared with controls (Figure 3B; $p=0.034$ ). Moreover, anti-IL-23(p19) treatment decreased insulitis severity (Figure 3A). These data suggest that inhibition of inflammatory pathways identified by functional genomic analysis of human islets from individuals with T1D can alter the course of disease in NOD mice. 


\section{Discussion}

Therapeutic interventions derived from pathways first identified to be altered in rodent disease have not effectively translated to interventions in human T1D. We postulate this is because targets are selected based on a disease pathophysiology specific to NOD mice, which may be different from that of the human disease. Therefore, we proposed the alternative concept of selecting targets for subsequent studies in animal models by first examining the relevant human tissue, in this case pancreatic islets from individuals with T1D. Thus, pancreatic islets from two individuals with T1D, where disease duration was vastly different, were analyzed. Specifically, we performed functional genomic analysis of islets from donors with short-duration disease (3 years) or long-duration disease (27 years). When compared to islets from non-diabetic donors, our analysis identified pathways that displayed distinctly different expression patterns between the islets of the two T1D donors and between the islets from the T1D donors and nondiabetic controls. This identified, among others, a pathway governed by IL-23(p19), which was selected for further study in NOD mice. We recognize that there are caveats associated with the study of only two T1D donors. On the other hand, the treatment of NOD mice early in disease progression with an inhibitor for one of these altered human pathways resulted in a significant reduction in disease incidence. Therefore, we believe that the present study underscores the value of using human tissue as the primary point of interrogation, as well as the utility of mouse models to then manipulate these human disease-relevant pathways to support possible therapeutic translation. 
One of the most distinct differences observed in the data was the upregulation of innate immunity-related pathways in T1D islets, particularly in short-duration disease. This finding is in line with previously published histology from pancreata of individuals with $\mathrm{T}_{1} \mathrm{D}^{21}$. Our data are also in line with flow cytometry analysis of islets from the same individual with short-duration T1D (nPOD69), which were subjected to a T cell stimulatory assay that confirmed the presence of CD45+ cells in the islets ${ }^{22}$. Given previous reports that insulitis in human T1D is not uniform and challenging to observe ${ }^{7,8}$, an additional interpretation of our data is that the islets themselves express certain genes, particularly chemokines, which influence the recruitment of inflammatory cells to the pancreas early in disease. Given the striking similarity in expression patterns observed in short-duration T1D, which showed more active inflammation, with previous findings in islets exposed to IL-1 beta and IFN-gamma ${ }^{18}$, it is conceivable that these, or other cytokines signaling via similar pathways, are in the islet environment and relevant for early stage human disease pathogenesis. These observations are supported by previous studies that identified an "interferon signature" in islets from pancreas biopsies of patients soon after diabetes diagnosis ${ }^{23}$. Another significant finding was the increased IL23A in short-duration T1D. Th17expressing T cells may participate in human T1D progression ${ }^{24-26}$. IL-23 augments Th17 cell development and interestingly, IL-23 shares the p40 subunit with IL-12 but pairs with a $19 \mathrm{kDa}$ peptide (p19). Similar to IL-12, IL-23 is secreted by activated APCs. Early studies documenting the role of IL-12 in Th1-mediated disease manipulated the p40 subunit, which is shared between IL-12 and IL-23. Therefore, p40 manipulation likely impacted both Th1 and Th17 pathways. Our 
identification of increased $I L 23 A(\mathrm{p} 19)$ suggested that manipulating this factor, rather than $\mathrm{p} 40$, may be useful to dampen the upregulation of inflammatory pathways early in disease.

Interestingly, an IL23A haplotype variant was identified to have a protective effect on T1D ${ }^{27}$, which implicates a role for this target in autoimmune diabetes. Multiple active clinical trials evaluating Mirikizumab (NCT01947933, NCT02891226, NCT02899988, and NCT02589665), which targets IL23(p19), as a therapeutic for other autoimmune-related indications further supported the evaluation of this target in reducing the inflammatory response and therefore possibly T1D progression. Our treatment of NOD mice with anti-IL23(p19) showed this effectintervention early in disease progression in NOD mice can significantly influence pathogenesis. We recognize that using NOD mice permits the manipulation of a target or pathways earlier in the disease progression than would be possible in humans, as interventions in humans can only occur in most cases after the disease is diagnosed. However, identifying an effect at this early stage of disease progression in NOD mice suggests that additional studies to determine the effect at later stages of disease progression in NOD mice are warranted. Ultimately, our study provides proof of concept that direct analysis of human islets is valuable for identifying promising targets for effective putative therapeutic interventions in human T1D. Moreover, studies of human disease tissue can lead to the repurposing of developed drugs or biologicals thereby speeding progress toward effective human therapeutics for T1D.

\section{Acknowledgments:}


We would like to thank Janssen Research and Development, LLC for providing reagents (CNTO 6163 and CNTO1322), Dr. Farooq Syed for assistance with human islet culture and preparation, and members of the Mastracci lab for helpful discussions. Funding for this study was provided by a Juvenile Diabetes Research Foundation Career Development Award to TLM (5-CDA-2016194-A-N), a National Institutes of Health Human Islet Research Network grant to RGM and DLE (UC4 DK104166) and a Juvenile Diabetes Research Foundation grant to RGM and MDP (17-2010-424). This research was performed with the support of the Network for Pancreatic Organ Donors with Diabetes (nPOD), a collaborative type 1 diabetes research project sponsored by JDRF and the Helmsley Charitable Trust. Organ Procurement Organizations (OPO) partnering with nPOD to provide research resources are listed at http://www.jdrfnpod.org/forpartners/npod-partners/.

\section{References:}

1. Op de Beeck A, Eizirik DL. Viral infections in type 1 diabetes mellitus--why the ${ }^{2}$ cells? Nat Rev Endocrinol. 2016;12(5):263-273. doi:10.1038/nrendo.2016.30

2. Anderson MS, Bluestone JA. The NOD mouse: a model of immune dysregulation. Annu Rev Immunol. 2005;23:447-485. doi:10.1146/annurev.immunol.23.021704.115643

3. Colvin SC, Maier B, Morris DL, Tersey SA, Mirmira RG. Deoxyhypusine synthase promotes differentiation and proliferation of T helper type 1 (Th1) cells in autoimmune diabetes. J Biol Chem. 2013;288(51):36226-36235. doi:10.1074/jbc.M113.473942

4. Tersey SA, Colvin SC, Maier B, Mirmira RG. Protective effects of polyamine depletion in mouse models of type 1 diabetes: implications for therapy. Amino Acids. 2014;46(3):633-642. doi:10.1007/s00726-013-1560-7

5. Matthews JB, Staeva TP, Bernstein PL, Peakman M, von Herrath M, ITN-JDRF Type 1 Diabetes Combination Therapy Assessment Group. Developing combination immunotherapies for type 1 diabetes: recommendations from the ITN-JDRF Type 1 Diabetes Combination Therapy Assessment Group. Clin Exp Immunol. 2010;160(2):176-184. doi:10.1111/j.13652249.2010.04153.x 
6. Simmons KM, Gottlieb PA, Michels AW. Immune Intervention and Preservation of Pancreatic Beta Cell Function in Type 1 Diabetes. Curr Diab Rep. 2016;16(10):97.

doi:10.1007/s11892-016-0793-8

7. In't Veld P. Insulitis in human type 1 diabetes: a comparison between patients and animal models. Semin Immunopathol. 2014;36(5):569-579. doi:10.1007/s00281-014-0438-4

8. Campbell-Thompson M, Fu A, Kaddis JS, et al. Insulitis and ${ }^{2}$-Cell Mass in the Natural History of Type 1 Diabetes. Diabetes. 2016;65(3):719-731. doi:10.2337/db15-0779

9. Atkinson MA, Bluestone JA, Eisenbarth GS, et al. How does type 1 diabetes develop?: the notion of homicide or ${ }^{2}$-cell suicide revisited. Diabetes. 2011;60:1370-1379. doi:10.2337/db10-1797

10. Eizirik DL, Colli ML, Ortis F. The role of inflammation in insulitis and beta-cell loss in type 1 diabetes. Nat Rev Endocrinol. 2009;5(4):219-226. doi:10.1038/nrendo.2009.21

11. Maganti A, Evans-Molina C, Mirmira R. From immunobiology to ${ }^{2}$-cell biology: the changing perspective on type 1 diabetes. Islets. 2014;6(2):e28778. doi:10.4161/isl.28778

12. Kim D, Pertea G, Trapnell C, Pimentel H, Kelley R, Salzberg SL. TopHat2: accurate alignment of transcriptomes in the presence of insertions, deletions and gene fusions. Genome Biol. 2013;14(4):R36. doi:10.1186/gb-2013-14-4-r36

13. Trapnell C, Pachter L, Salzberg SL. TopHat: discovering splice junctions with RNA-Seq. Bioinforma Oxf Engl. 2009;25(9):1105-1111. doi:10.1093/bioinformatics/btp120

14. Harrow J, Frankish A, Gonzalez JM, et al. GENCODE: the reference human genome annotation for The ENCODE Project. Genome Res. 2012;22(9):1760-1774.

doi:10.1101/gr.135350.111

15. Harrow J, Denoeud F, Frankish A, et al. GENCODE: producing a reference annotation for ENCODE. Genome Biol. 2006;7 Suppl 1:S4.1-9. doi:10.1186/gb-2006-7-s1-s4

16. Montgomery SB, Sammeth M, Gutierrez-Arcelus M, et al. Transcriptome genetics using second generation sequencing in a Caucasian population. Nature. 2010;464(7289):773-777. doi:10.1038/nature08903

17. Mortazavi A, Williams BA, McCue K, Schaeffer L, Wold B. Mapping and quantifying mammalian transcriptomes by RNA-Seq. Nat Methods. 2008;5(7):621-628.

doi:10.1038/nmeth.1226

18. Eizirik DL, Sammeth M, Bouckenooghe T, et al. The human pancreatic islet transcriptome: expression of candidate genes for type 1 diabetes and the impact of proinflammatory cytokines. PLoS Genet. 2012;8(3):e1002552. doi:10.1371/journal.pgen.1002552

19. Mastracci TL, Anderson KR, Papizan JB, Sussel L. Regulation of Neurod1 contributes to the lineage potential of Neurogenin3+ endocrine precursor cells in the pancreas. PLoS Genet. 2013;9(2):e1003278. doi:10.1371/journal.pgen.1003278

20. Cabrera SM, Colvin SC, Tersey SA, Maier B, Nadler JL, Mirmira RG. Effects of Combination Therapy with Dipeptidyl Peptidase-IV and Histone Deacetylase Inhibitors in the NOD Mouse Model of Type 1 Diabetes. Clin Exp Immunol. 2013;172:375-382. doi:10.1111/cei.12068 
21. Martino L, Masini M, Bugliani M, et al. Mast cells infiltrate pancreatic islets in human type 1 diabetes. Diabetologia. 2015;58(11):2554-2562. doi:10.1007/s00125-015-3734-1

22. Babon JAB, DeNicola ME, Blodgett DM, et al. Analysis of self-antigen specificity of islet-infiltrating T cells from human donors with type 1 diabetes. Nat Med. 2016;22(12):14821487. doi: $10.1038 / \mathrm{nm} .4203$

23. Lundberg M, Krogvold L, Kuric E, Dahl-Jørgensen K, Skog O. Expression of InterferonStimulated Genes in Insulitic Pancreatic Islets of Patients Recently Diagnosed With Type 1 Diabetes. Diabetes. 2016;65(10):3104-3110. doi:10.2337/db16-0616

24. Arif S, Moore F, Marks K, et al. Peripheral and islet interleukin-17 pathway activation characterizes human autoimmune diabetes and promotes cytokine-mediated ${ }^{2}$-cell death. Diabetes. 2011;60(8):2112-2119. doi:10.2337/db10-1643

25. Emamaullee JA, Davis J, Merani S, et al. Inhibition of Th17 cells regulates autoimmune diabetes in NOD mice. Diabetes. 2009;58(6):1302-1311. doi:10.2337/db08-1113

26. Ochs HD, Oukka M, Torgerson TR. TH17 cells and regulatory T cells in primary immunodeficiency diseases. J Allergy Clin Immunol. 2009;123(5):977-983; quiz 984-985. doi:10.1016/j.jaci.2009.03.030

27. Costa VS, Santos AS, Fukui RT, Mattana TCC, Matioli SR, Silva MER. Protective effect of interleukin-23A (IL23A) haplotype variants on type 1A diabetes mellitus in a Brazilian population. Cytokine. 2013;62(2):327-333. doi:10.1016/j.cyto.2013.03.015

\section{Figure Legends}

Figure 1. Cluster analysis of RNA sequencing data from human tissue samples including human islets with T1D. Gene expression levels were compared for the five islet preparations (two T1D and three controls) and 16 background tissues from the Illumina Human Body Map (GEO accession GSE30611). Genes with expression of RPKM $>0.5$ in at least one of the samples, and with potential pathogenic relevance, were considered for analysis. For each pair of samples, a Spearman correlation coefficient (SCC) was computed from the RPKM expression levels. The heatmap shows the clustering dendrograms inferred by employing $(1-\mathrm{SCC})$ as distance function and complete linkage as clustering function, showing both a tight cluster of the 
five islet preparations against the background tissues on one hand and a tight cluster of the two T1D samples as compared to the three normoglycemic control samples.

Figure 2. Ingenuity Pathway Analysis (IPA) for up-regulated genes in canonical pathways identified in short-duration T1D. 667 genes were considered up-regulated in the short duration T1D sample compared to the normoglycemic control donors. These genes were subjected to gene set enrichment analysis using the IPA database based on Benjamini-Hochberg corrected Fisher tests. The "canonical pathways" identified by IPA for the upregulated genes in short-duration T1D are displayed. The length of the blue bars indicates the significance of the association between the set of genes and the keyword, and is expressed as minus the logarithm of the probability that a random set of genes from the human genome would be associated with the same keyword. The straight orange line indicates a threshold of 0.05 (corresponding to a $-\log$ (B-H p-value) of 1.3 on the upper Y-axis). The orange dots for each pathway (connected with a line) indicate the ratio (lower Y-axis) between the number of genes observed in the data set and the total number of genes in the pathway (as annotated in IPA).

Figure 3. Diabetes free survival of NOD mice treated with or without IL-23/p19 inhibitor. (A) Insulitis scoring for NOD mice 6 weeks of age treated with CNTO6163 to block IL23/p19 or isotype control. (B) NOD mice treated with CNTO6163 to block IL23/p19 showed significantly 
reduced diabetes incidence compared with isotype control treated NOD mice of the same age ( $p$ $=0.034)$. 


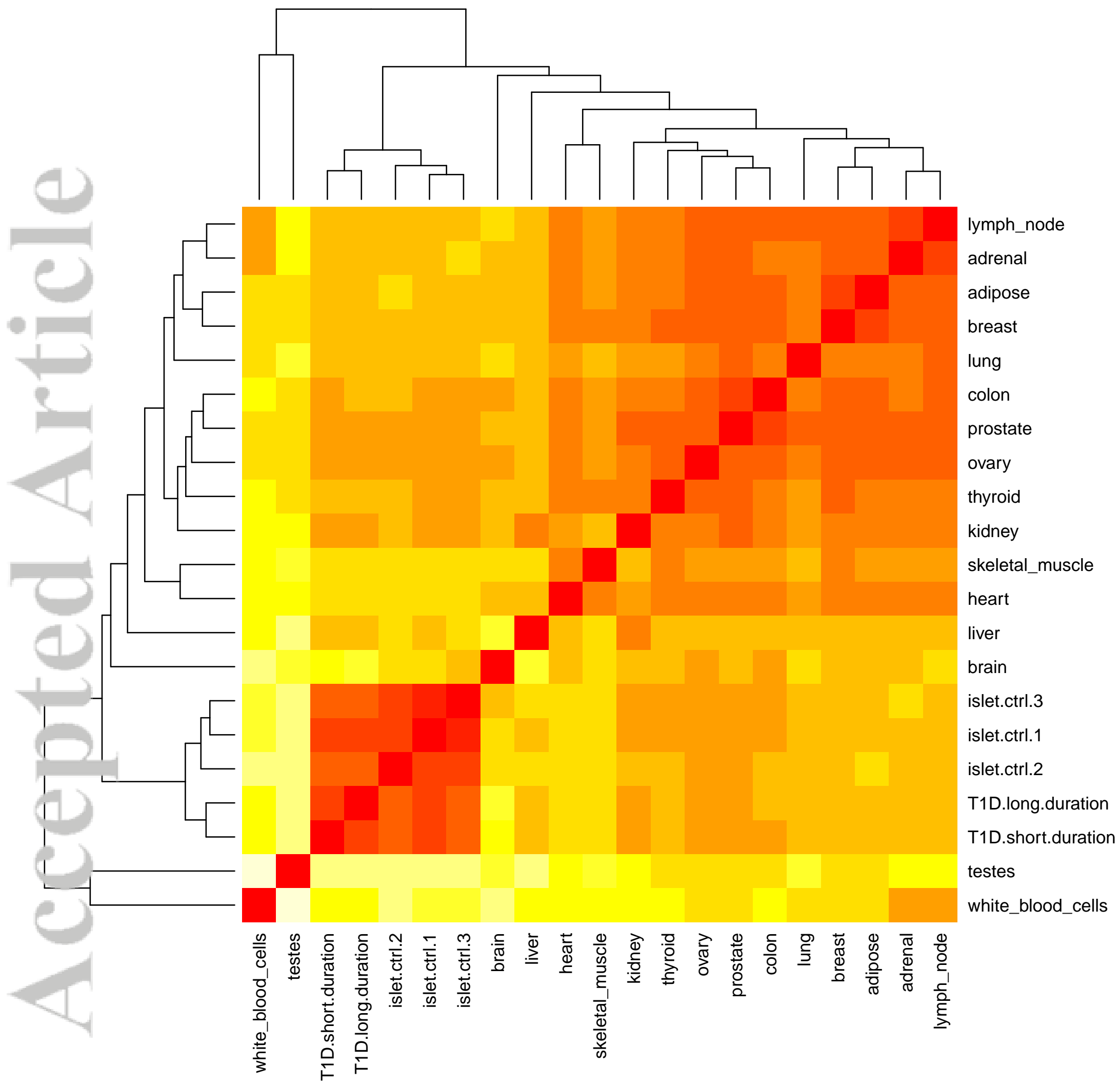

This article is protected by copyright. All rights reserved. 


\section{Granulocyte Adhesion and Diapedesis}

Agranulocyte Adhesion and Diapedesis

\section{LXR/RXR Activation}

Role of Macrophages, Fibroblasts and Endothelial Cells in Rheumatoid Arthritis

es in Mediating Communication between Immune Cells

\section{Comm on between Innate and Adaptive Immune Cells}

Altered T Cell and B Cell Signaling in Rheumatoid Arthritis

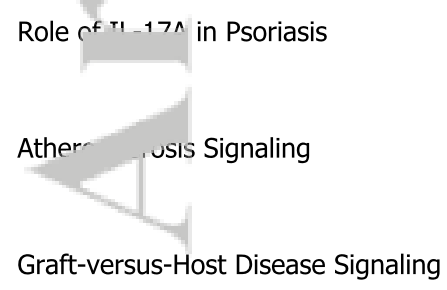

Differential Regulation of Cytokine Production in Intestinal Epithelial Cells by IL-17A and IL-17F

Acute Phase esponse Signaling

TREM1 Signaling

Dend ration

Hepatic _..viestasis

Toll-like keceptor Signaling

Crosstalk between Dendritic Cells and Natural Killer Cells

Hepatic Fibrosis / Hepatic Stellate Cell Activation

Role of Hypercytokinemia/hyperchemokinemia in the Pathogenesis of Influenza

Role of IL-17A in Arthritis

TNFR2 Signaling
- $\log (\mathrm{B}-\mathrm{H}$ p-value)

\begin{tabular}{|l|l|l|l|l|l|}
\hline & & & & & \\
\hline
\end{tabular}
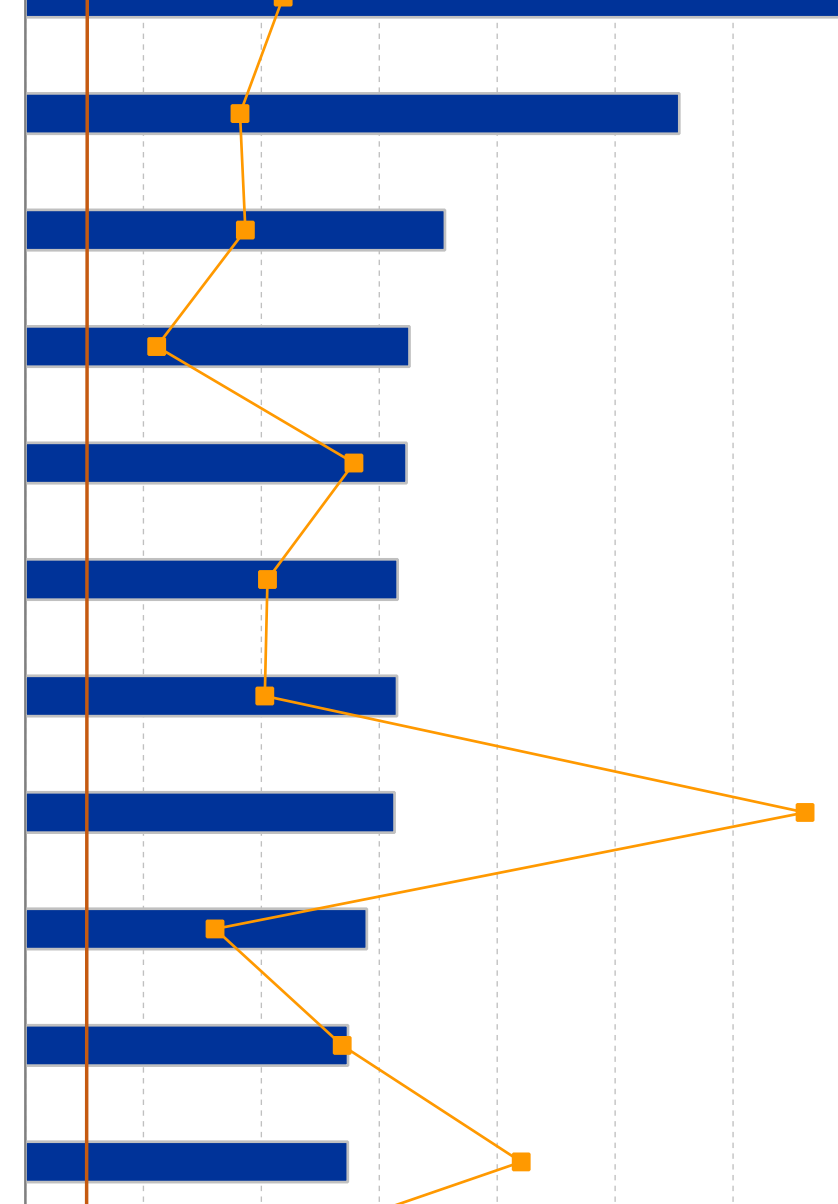

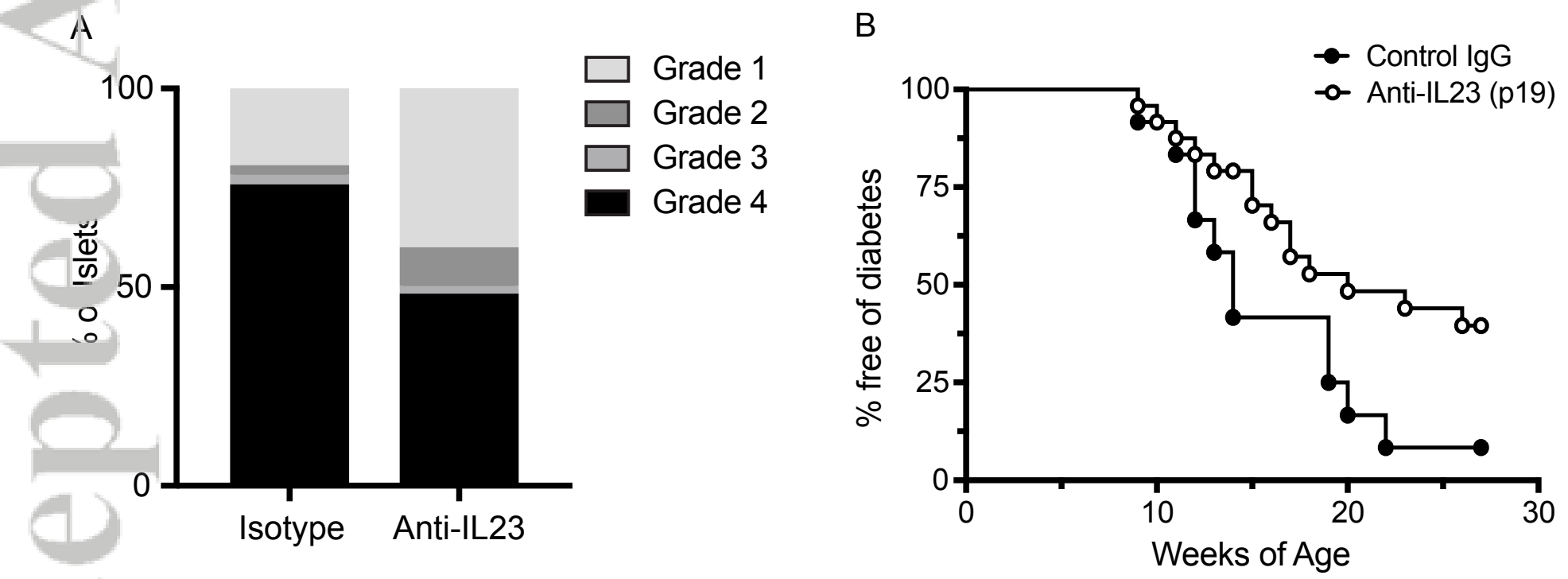

Figure 3. Mastracci TL., et al.

This article is protected by copyright. All rights reserved. 
Table 1. Expression of selected genes in human donor islets from short-duration (SD) T1D, long-duration (LD) T1D and non-diabetic controls. Genes with expression of RPKM $>0.5$ in at least one of the samples studied and with potential pathogenic relevance are shown. For key cytokines or other immune-relevant signaling molecules (e.g. IFNG, IL17A etc) values are shown even when below detection level at all samples.

\begin{tabular}{|c|c|c|c|c|}
\hline Gene & Description & SD-T1D & LD-T1D & Ctrl-median \\
\hline ANK3 & ankyrin 3, node of Ranvier (ankyrin G) & 3.864 & 5.586 & 4.192 \\
\hline CCL5 & chemokine (C-C motif) ligand 5 & 8.591 & 0.877 & 0.707 \\
\hline CCR5 & C-C motif chemokine receptor 5 & 0.175 & 0.103 & 0.103 \\
\hline CD2 & CD2 molecule & 0 & 0.260 & 0.034 \\
\hline$C D 4$ & CD4 molecule & 0.625 & 0.290 & 3.473 \\
\hline CD5 & CD5 molecule & 0 & 0.072 & 2.636 \\
\hline CD6 & CD6 molecule & 0 & 0.359 & 0.660 \\
\hline CD8A & CD8a molecule & 0.134 & 0.233 & 0.280 \\
\hline CD14 & CD14 molecule & 12.542 & 2.371 & 58.727 \\
\hline CD22 & CD22 molecule & 0.505 & 0 & 4.567 \\
\hline CD68 & CD68 molecule & 475.127 & 155.884 & 216.451 \\
\hline CD86 & CD86 molecule & 1.631 & 0.162 & 0.537 \\
\hline СЕВРВ & $\begin{array}{l}\text { CCAAT/enhancer binding protein } \\
(\mathrm{C} / \mathrm{EBP}) \text {, beta }\end{array}$ & 22.271 & 15.710 & 9.911 \\
\hline CLEC5A & ENSG00000258227 & 0.108 & 0.273 & 0.372 \\
\hline CSF $2 R B$ & $\begin{array}{l}\text { colony stimulating factor } 2 \text { receptor, } \\
\text { beta, low-affinity (granulocyte- } \\
\text { macrophage) }\end{array}$ & 2.536 & 0.134 & 0.908 \\
\hline CSF3 & colony stimulating factor 3 (granulocyte) & 253.810 & 1.265 & 2.582 \\
\hline CTSB & cathepsin B & 203.303 & 163.176 & 433.834 \\
\hline CTSH & cathepsin $\mathrm{H}$ & 42.622 & 165.632 & 52.752 \\
\hline CXCL1 & $\begin{array}{l}\text { chemokine (C-X-C motif) ligand } 1 \\
\text { (melanoma growth stimulating activity, } \\
\text { alpha) }\end{array}$ & 1379.470 & 67.349 & 152.284 \\
\hline CXCL2 & chemokine (C-X-C motif) ligand 2 & 443.036 & 84.223 & 90.415 \\
\hline CXCL3 & chemokine (C-X-C motif) ligand 3 & 164.310 & 13.695 & 15.896 \\
\hline CXCL6 & $\begin{array}{l}\text { chemokine (C-X-C motif) ligand } 6 \\
\text { (granulocyte chemotactic protein } 2 \text { ) }\end{array}$ & 373.120 & 92.100 & 18.942 \\
\hline CXCL9 & chemokine (C-X-C motif) ligand 9 & 0.214 & 0.031 & 0.023 \\
\hline CXCL10 & chemokine (C-X-C motif) ligand 10 & 0.443 & 0.241 & 0.075 \\
\hline EBF1 & early B-cell factor 1 & 1.181 & 0.264 & 1.088 \\
\hline EMR2 & $\begin{array}{l}\text { egf-like module containing, mucin-like, } \\
\text { hormone receptor-like } 2\end{array}$ & 1.090 & 0.342 & 0.417 \\
\hline FCGRT & $\begin{array}{l}\text { Fc fragment of IgG, receptor, } \\
\text { transporter, alpha }\end{array}$ & 42.974 & 42.853 & 90.638 \\
\hline GATAЗ & GATA binding protein 3 & 0 & 0 & 0.042 \\
\hline
\end{tabular}




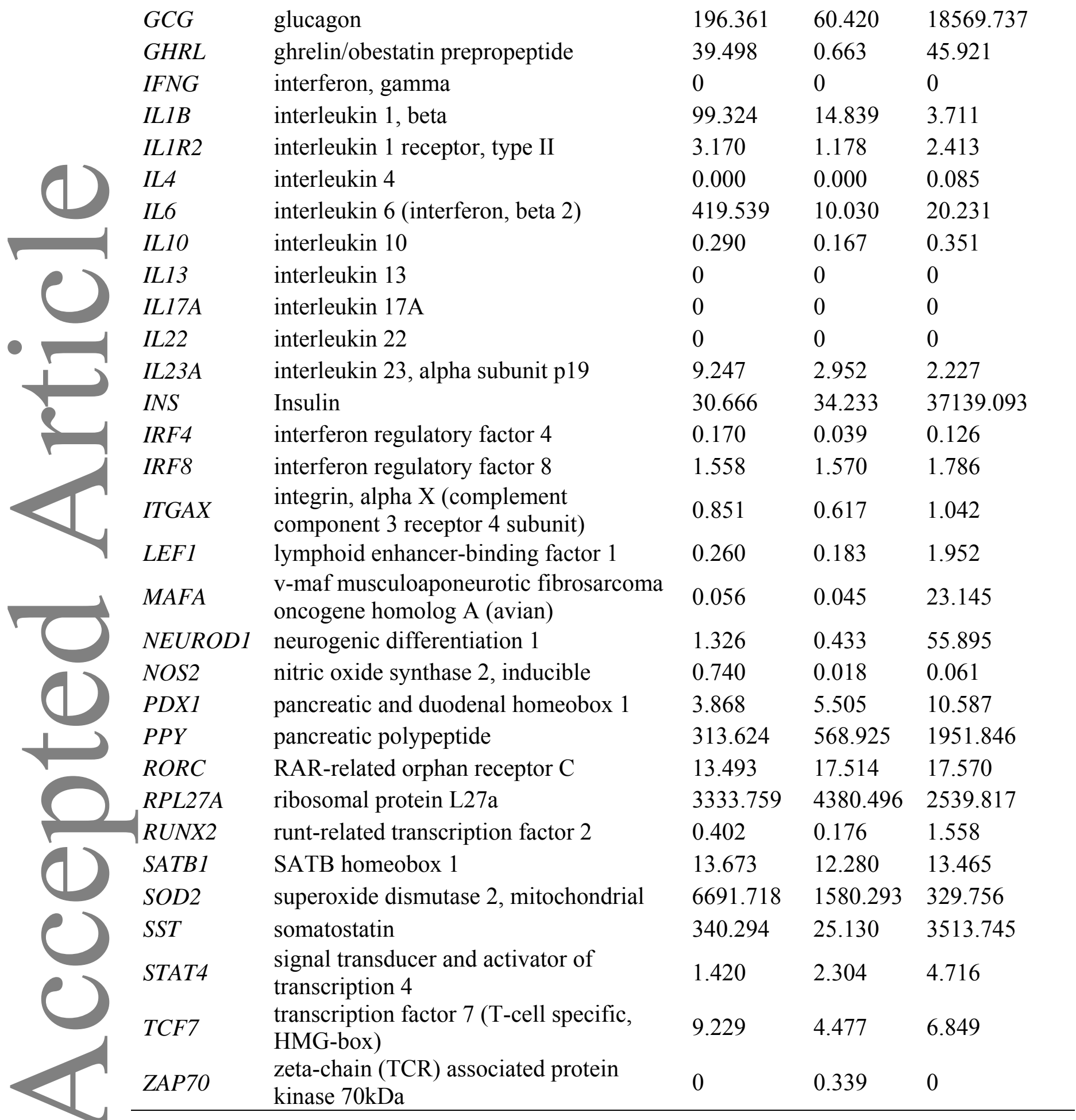


Table 2. Up- and down-regulated pathways identified in short- and long-duration T1D islets. Lists are restricted to Top 10 pathways identified for each group. The complete list for each group with additional detail can be found in the Supplemental Materials. The BenjaminiHochberg adjusted p-value for the significance (-Log10 (B-H p-value) used a cutoff of 1.3, which corresponds to $>-\log 10(\mathrm{~B}-\mathrm{H} \mathrm{p}$-value $=0.05)$. The ratio refers to the number of genes in the dataset compared to the total number of genes in the corresponding pathway.

\begin{tabular}{lcc}
\hline \multicolumn{1}{c}{ Up-regulated Pathways: Short-duration T1D } & -log(B-H p-value) & Ratio \\
\hline Granulocyte Adhesion and Diapedesis & 18.3 & 0.203 \\
Agranulocyte Adhesion and Diapedesis & 13.9 & 0.169 \\
LXR/RXR Activation & 8.89 & 0.174 \\
Role of Macrophages, Fibroblasts and Endothelial Cells in RA & 8.14 & 0.104 \\
Role of Cytokines in Mediating Communication between & & \\
Immune Cells & 8.08 & 0.259 \\
Communication between Innate and Adaptive Immune Cells & 7.89 & 0.191 \\
Altered T Cell and B Cell Signaling in Rheumatoid Arthritis & 7.88 & 0.189 \\
Role of IL-17A in Psoriasis & 7.82 & 0.615 \\
Atherosclerosis Signaling & 7.24 & 0.15 \\
Graft-versus-Host Disease Signaling & 6.84 & 0.25 \\
\end{tabular}

Up-regulated Pathways: Long-duration T1D

$\gamma$-glutamyl Cycle

2.56

0.312

Xenobiotic Metabolism Signaling

2.56

0.0662

Acute Phase Response Signaling

2.56

0.0828

Glutathione-mediated Detoxification

2.5

0.207

FXR/RXR Activation

2.2

0.0873

Eicosanoid Signaling

2.2

0.119

LPS/IL-1 Mediated Inhibition of RXR Function

2.2

0.0676

Complement System

2.17

0.162

Leukotriene Biosynthesis

2.08

0.286

Aryl Hydrocarbon Receptor Signaling

2

0.0786

\section{Down-regulated Pathways: Short-duration T1D}

GPCR-Mediated Nutrient Sensing in Enteroendocrine Cells

0.271

Cardiac $\beta$-adrenergic Signaling

3.24

0.212

GPCR-Mediated Integration of Enteroendocrine Signaling

2.54

0.25

Axonal Guidance Signaling

2.42

0.14

Cellular Effects of Sildenafil (Viagra)

2.27

0.192

Dopamine-DARPP32 Feedback in cAMP Signaling

2.27

0.179 
Signaling by Rho Family GTPases

Breast Cancer Regulation by Stathmin1

CDK5 Signaling

2.08

0.202

Maturity Onset Diabetes of Young (MODY) Signaling

2.08

0.381

Down-regulated Pathways: Long-duration T1D

Axonal Guidance Signaling

3.25

0.156

GPCR-Mediated Nutrient Sensing in Enteroendocrine Cells

3.25

0.259

Hepatic Fibrosis / Hepatic Stellate Cell Activation

2.95

0.191

LXR/RXR Activation

2.83

0.215

GPCR-Mediated Integration of Enteroendocrine Signaling

2.53

0.25

Neuropathic Pain Signaling In Dorsal Horn Neurons

2.53

0.211

Cardiac $\beta$-adrenergic Signaling

2.53

0.197

G-Protein Coupled Receptor Signaling

1.99

0.154

Regulation of the Epithelial-Mesenchymal Transition Pathway

1.99

0.169

Th2 Pathway

1.99

0.18

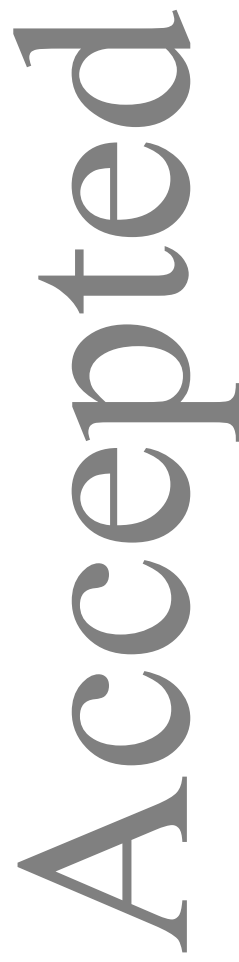

